\title{
Investigation of the potential role of preoperative chemotherapy in treatment for gastric cancer with outlet obstruction
}

\author{
XUELONG JIAO and YANBING ZHOU \\ Department of General Surgery, The Affiliated Hospital of Qingdao University, Qingdao, Shandong 266003, P.R. China
}

Received February 6, 2015; Accepted June 19, 2015

DOI: $10.3892 / \mathrm{mco} .2015 .587$

\begin{abstract}
Preoperative chemotherapy is currently recognized as the recommended treatment for advanced gastric cancer. Whether there is a role of preoperative chemotherapy in the treatment for advanced gastric cancer with gastric outlet obstruction (GOO) is unknown. In order to explore the potential feasibility of preoperative chemotherapy for advanced gastric cancer with GOO, and to encourage the probe into optimal treatment strategies for advanced gastric cancer with GOO in the current era of preoperative chemotherapy prevailing, a systematic literature search was conducted with a multistage process. The characteristics of the retrieved publications were summarized and the essential information was extracted. Only 11 studies associated with preoperative chemotherapy for advanced gastric cancer with GOO were identified. Among them, 9 were case reports, while the other 2 were research reports of retrospective studies. None were of prospective studies. The paucity of the literature in this field is a marked finding of the present study, which reports the emerging attempts at preoperative chemotherapy for advanced gastric cancer patients with GOO, as no high-quality data are available. The definite role of chemotherapy as an initial treatment for advanced gastric cancer patients with GOO remains unclear. Clinical trials are expected to be conducted in order to explore the feasibility, safety and efficacy of preoperative chemotherapy for advanced gastric cancer patients with GOO.
\end{abstract}

\section{Introduction}

Large randomized trials $(1,2)$ have demonstrated certain clinical benefits of preoperative therapy for gastric cancer. Subsequently, the role of preoperative chemotherapy has been established in locally advanced gastric cancer patients.

Correspondence to: Professor Yanbing Zhou, Department of General Surgery, The Affiliated Hospital of Qingdao University, 16 Jiangsu Road, Qingdao, Shandong 266003, P.R. China

E-mail: zhouyanbing999@aliyun.com

Key words: stomach neoplasm, gastric outlet obstruction, pyloric stenosis, neoadjuvant therapy, preoperative chemotherapy, nutritional support
However, such patients complicated with gastric outlet obstruction (GOO) are generally not treated as appropriate candidates for preoperative chemotherapy in the current clinical practice due to patients' intolerance to chemotherapeutic medications. Whether there is a role of chemotherapy as a preoperative treatment for advanced gastric cancer with GOO is unknown.

Whether GOO had been considered as eligibility or ineligibility criterion of protocols was not disclosed in previously published literature regarding clinical trials, even in the MAGIC (1) and FNCLCC ACCORD 07-FFCD 9703 (2) studies that are landmarks in this field. Thus, there is no available data regarding feasibility, safety or efficacy of preoperative chemotherapy for the subgroup of gastric cancer patients with GOO revealed in these studies. Additionally, GOO is occasionally treated as an exclusive criterion in the protocol of certain clinical trials concerning preoperative chemotherapy for gastric cancer (3). Therefore, the universality of clinical benefit induced by preoperative chemotherapy has some limitations due to the lack of data in the GOO subset, which is an elementary subgroup of gastric cancer.

Whether there is a potential role of preoperative chemotherapy in the treatment of gastric cancer patients with GOO requires investigation. In order to answer this novel question, a literature review was conducted and a prospective outlook was made in this field.

\section{Materials and methods}

Search strategies. A systematic electronic literature search was conducted in the PubMed database, Embase and Cochrane Library (until January 2015). All publications associated with preoperative chemotherapy for gastric cancer patients with GOO were targets of the retrieval. Clinical trials registered in NIH (ClinicalTrials.gov) were also retrieved. No restrictions on language were applied to the retrieval strategies. Retrieval with a multistage process was carried out as follows.

Step 1. In the PubMed database, the search was conducted using terms of medical subject headings (MeSH) that were 'stomach neoplasms', ('gastric outlet obstruction' or 'pyloric stenosis') and 'neoadjuvant therapy', which was firstly applied.

Step 2. Due to the extreme scarcity of the retrieved results, an additional extensive search using free terms was further conducted.

The equivalent free terms of 'stomach neoplasms' are 'gastric cancer', 'gastric carcinoma', 'gastric adenocarcinoma', 
'stomach cancer', 'stomach carcinoma', 'stomach adenocarcinoma', 'cancer of the stomach', 'carcinoma of the stomach', 'adenocarcinoma of the stomach', 'gastric neoplasm', 'gastric neoplasms', 'neoplasm of the stomach' and 'neoplasms of the stomach'.

The equivalent free terms of 'gastric outlet obstruction' and 'pyloric stenosis' are 'pyloric obstruction', 'pyloric stenosis', 'antrum obstruction', 'antrum stenosis', 'gastric obstruction', 'gastric stenosis', 'outlet obstruction', 'outlet stenosis', 'obstruction of the stomach', 'obstruction of the pylorus', 'obstruction of the antrum' and 'stenosis of the pylorus'.

The equivalent free terms of 'neoadjuvant therapy' are 'neoadjuvant chemotherapy', 'neoadjuvant treatment', 'preoperative therapy', 'preoperative chemotherapy', 'preoperative treatment', 'perioperative therapy', 'perioperative chemotherapy', 'perioperative treatment' and 'induction chemotherapy'.

Step 3. The MeSH search (step 1) was extended by substituting 'drug therapy' for 'neoadjuvant therapy'.

Step 4. Similar retrieval strategies, as mentioned above, were also conducted in Embase and Cochrane Library.

Step 5. Retrieval in ClinicalTrials.gov was performed in 'expert search' using the free terms mentioned above.

Data extraction. All the studies obtained were read in full. Characteristics of the retrieved studies were summarized, and the essential information of each eligible study was extracted, such as author names, year of publication, country of investigators, sample size, chemotherapy regimen, cycles of preoperative chemotherapy, clinical outcomes and adverse events. Two reviewers independently extracted the data from each study. No statistical analysis was employed on account of limited quantity and quality of these publications.

\section{Results}

Application of MeSH terms. The search using the MeSH terms in step 1 located only 2 studies, of which 1 was in accordance with the retrieval purpose (4). A further search using free and MeSH terms located 20 studies, of which 11 met the retrieval purpose (4-14) (Step 2). Among these, 2 were clinical research studies $(4,5)$, while the other 9 were case reports (6-14). No additional literature was obtained either by substituting 'drug therapy' for 'neoadjuvant therapy' (step 3) or from Embase and Cochrane Library (step 4). No relevant trial registered in ClinicalTrials.gov met the criteria (step 5). Detailed characteristics regarding the 11 studies are summarized in Table I.

The 9 case reports involved 10 patients, whose age ranged from 44 to 74 years, with a male to female ratio of 6:4. All these individuals had a primary GOO caused by advanced gastric cancer and received preoperative chemotherapy with fluoropyrimidine-based regimens of 2-6 cycles. The chemicals concurrently administered with fluoropyrimidine varied from cisplatin $(7$ cases) $(6,7,9,11,13,14)$ to paclitaxel (4 cases) $(8,9,11,12)$, leucovorin (1 case) $(13)$ and methotrexate (1 case) (14). Trastuzumab was used in 3 HER2-positive patients $(6,7)$. Whether and how parenteral or enteral nutritional support was implemented was not disclosed in 8 (6,7,9-14) abstracts and 1 full text (8). Following preoperative chemotherapy, symptoms of GOO were relieved in
Table I. Distribution characteristics of the retrieved studies.

No. of retrieved

Distribution characteristics studies

Study classification

Clinical study

Case report

9

Nationality of the author

Eastern countries

China

2

Japan

9

Western countries

0

Publication journals

Chinese Journal of Cancer Research

Zhonghua Yi Xue Za Zhi

Gan to Kagaku Ryoho

1

Publication number in each decade

1990s

2

2000s

5

2010s

Language used in the original study

English

Chinese

1

Japanese

5 patients $(8-10,12,13)$, while they remained in 2 individuals $(11,14)$ and developed in 3 patients $(6,7)$. Details regarding the 10 subjects are shown in Table II.

The 2 clinical research studies were of retrospective studies performed in the Asian population. The essential information of the 2 studies are summarized in Table III and as follows.

A retrospective descriptive study. Yamaguchi et al (4) performed a critical evaluation of preoperative chemotherapy ('neoadjuvant chemotherapy' in the original text) with paclitaxel, fluorouracil and cisplatin for advanced gastric cancer patients complicated with GOO. Thirteen patients with far advanced or non-curative respectable gastric cancer with GOO received preoperative chemotherapy. These patients were treated with $40 \mathrm{mg} / \mathrm{m}^{2}$ paclitaxel on days 1 and 8 , combined with $6.5 \mathrm{mg} / \mathrm{m}^{2}$ cisplatin and $350 \mathrm{mg} / \mathrm{m}^{2}$ fluorouracil on days 1 through 8 followed by a 2 -week rest as one course. After at least two courses of treatment, the patients underwent gastrectomy with lymphadenectomy. The overall response rate (ORR) was $38.5 \%$. Seven patients had received staging laparoscopy prior to preoperative chemotherapy and 6 had free cancer cells in the peritoneal cavity. Of the 6 subjects with positive cytology at laparoscopy, 4 had no free cancer cells at surgery following preoperative chemotherapy. The median survival time was 405 days and the 1-year survival rate was $55.6 \%$. Toxicities were generally mild, and no serious adverse reactions were observed. There were only 2 cases that experienced grade 3 neutropenia. The authors concluded that preoperative chemotherapy with a combination of paclitaxel plus fluorouracil and cisplatin appeared to be an effective treatment for 


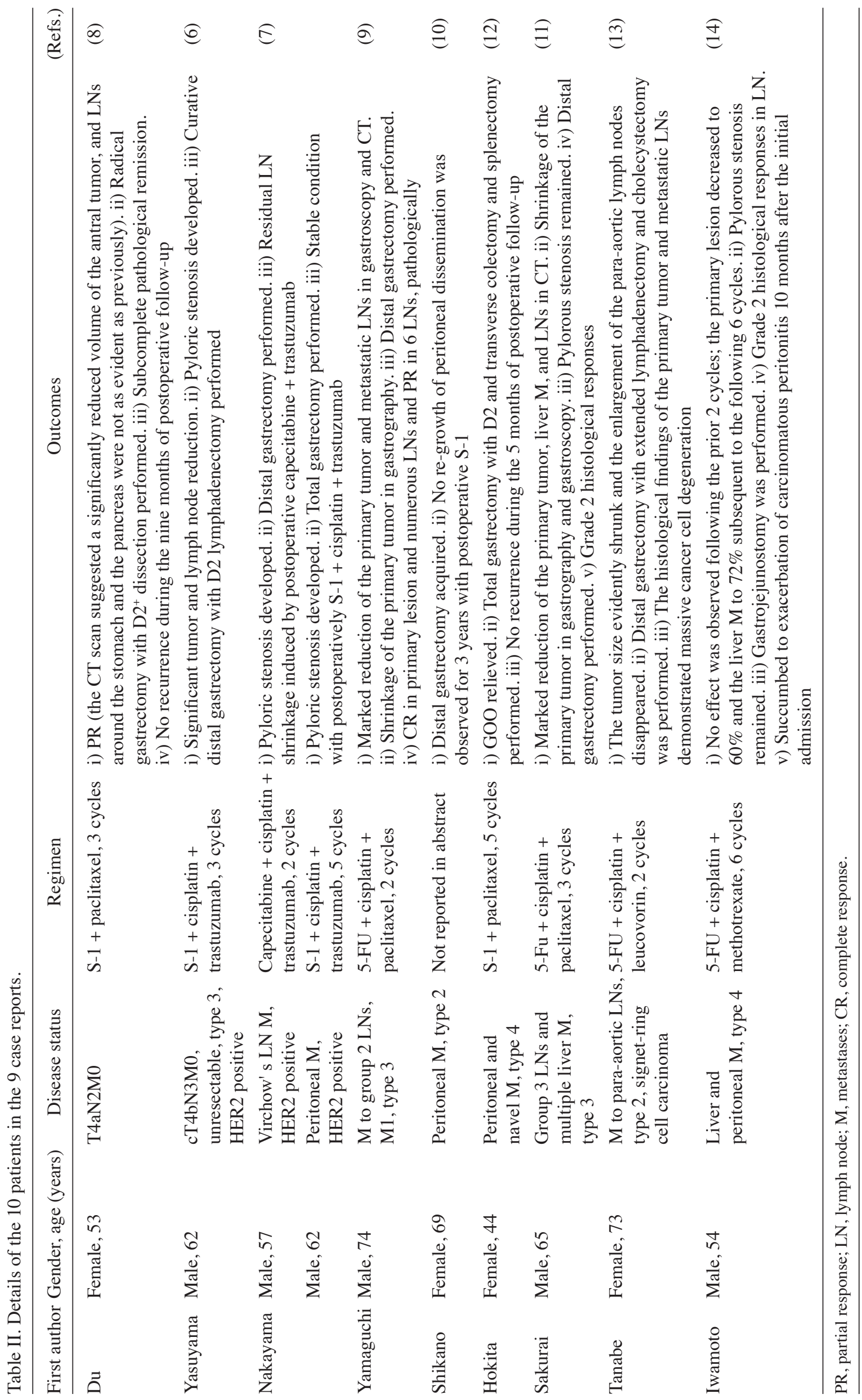


advanced gastric cancer patients with GOO. Whether and how parenteral or enteral nutritional support was implemented was not represented in the abstract. The data above were adapted from the online abstract.

A retrospective analytic study. Sun et al (5) conducted a retrospective study to explore the efficacies of preoperative chemotherapy ('neoadjuvant chemotherapy' in original text) plus nutritional support for advanced gastric cancer patients complicated with GOO. Retrospective analyses were performed for a total of 116 patients between January 2004 and June 2013. A total of 62 patients (group A) received preoperative chemotherapy (FOLFOX) plus concurrently administered nutritional support. Parenteral $(\mathrm{PN}, \mathrm{n}=30)$ or enteral $(\mathrm{EN}, \mathrm{n}=32)$ nutritional support was provided. The remaining 54 patients (group B) underwent exploratory laparotomy without preoperative chemotherapy. The serum level of albumin and quality of life score on the last preoperative day improved in group A. The extent of the improvement was more significant in the EN group compared with the PN group. ORR was 69.4\% (43/62) in group A [complete response (CR), 4; partial response (PR), 39; and stable disease (SD), 19], 84.4\% (27/32) in the EN group (CR, 3; PR, 24; and SD, 5) and 53.3\% (16/30) in the PN group (CR, 1; PR, 15; and SD, 14). The excision rate and the radical excision rate in group A (85.5 and $45.2 \%$ ) were significantly higher compared with group B $(64.8$ and $18.5 \%)(\mathrm{P}<0.05)$. No difference was observed in pathological response rates between groups A and B, but the grade II+III pathological response rate was higher in the EN group (12/32) compared with the PN group (3/30). No significant difference was observed in the postoperative morbidity rate between group A (22.6\%) and group B (33.3\%) ( $\mathrm{P}>0.05)$. The rate of postoperative gastric hypodynamics was higher in the PN group (10/30) compared with the EN group $(5 / 32)(\mathrm{P}<0.05)$. The authors concluded that nutritional support, particularly EN, could improve the nutritional status and quality of life in patients with gastric cancer complicated with GOO, and that preoperative chemotherapy plus nutritional support increased the rate of tumor excision. The information above was extracted from the full text of the original study written in Chinese.

\section{Discussion}

Gastric outlet obstruction, also known as pyloric obstruction, is defined as 'the clinical and pathophysiological consequence of any disease process that produces a mechanical impediment to gastric emptying' (Medscape) (15) or as 'the hindering of output from the stomach into the small intestine' (MeSH vocabulary thesaurus) (16), and is a frequently encountered impairment of gastric cancer patients in the clinical practice of medical and surgical oncologists. Individuals with GOO account for $31.6 \%$ of the gastric cancer patients (17).

Gastric cancer with GOO has a worse biological behavior. Chen et al (17) evaluated 551 gastric cancer patients with GOO and concluded that patients with this condition had a deeper cancer invasion and more lymph node metastases compared with those without. Similarly, Watanabe et al (18) conducted a clinicopathological study of gastric cancer with GOO, showing that these cancers were characterized by an infiltrating gross pattern and undifferentiated adenocarcinoma, and that these 
lesions showed a high incidence of serosal invasion, direct invasion into neighboring organs, peritoneal dissemination, lymph node metastasis and liver metastasis, as compared to the gastric cancers without GOO. These studies indicate that gastric cancers with GOO are generally in a more advanced stage.

Accordingly, gastric cancers with GOO have poor outcomes. Over two-thirds underwent palliative gastrojejunostomy without resection of the primary tumor or non-curative resection (18). The 5-year overall and disease-free survival rate of these patients were significantly lower compared with those without GOO (17). Thus, an improvement of the low curative resection rate and poor survival prognosis is required. As patients with GOO are in a more advanced stage, we hypothesize that this advanced condition may be a precise indication for preoperative chemotherapy.

However, the initial intervention for patients with GOO is more inclined to be surgical exploration instead of chemotherapy in clinical practice, past and present (18-26). For advanced gastric cancer patients, it is widely acknowledged that chemotherapy as an initial therapy is premised by the absence of 'passage disturbance' (24). Therefore, if there are GOO symptoms, chemotherapy is more likely to be applied postoperatively (24). Additionally, GOO is sometimes explicitly regarded as the exclusive criterion in the protocol of certain clinical trials concerning preoperative chemotherapy for gastric cancer (3). However, whether there is no possibility for the application of preoperative chemotherapy for gastric cancer patients with GOO remains to be explored. Therefore, whether dose chemotherapy as an initial intervention has potential clinical benefits equal to or different from surgery requires clarification. To explore this issue, the present literature review was conducted.

The multistage process and limited acquisition of the literature retrieval in the present study indicates that there is a modest number of medical practitioners implementing preoperative chemotherapy for gastric cancer patients with GOO. This may not be due to the unfeasibility of the mentioned treatment strategy, but due to the paucity of high-quality evidence to conclude whether chemotherapy as an initial treatment provided a clinical benefit that was equivalent to or better than that of surgery alone. However, certain valuable aspects can be extracted from these retrieved studies.

There was a potential feasibility of preoperative chemotherapy for GOO patients. Firstly, the 10 cases reported underwent chemotherapy for 2-6 cycles (6-14). The 13 individuals in the descriptive study (4) underwent gastrectomy following at least 2 cycles of drug therapy. The 62 subjects in the analytical study (5) received FOLFOX treatment by 2.7 cycles in average. These figures approximately met the number of preoperative chemotherapy cycles recommended in the NCCN guidelines (27). Secondly, of all the patients mentioned, only 2 cases suffering grade 3 neutropenia were reported with regards to the therapeutic toxicity (4), which indicated that the adverse effect of preoperative chemotherapy for GOO patients may be tolerable. Thirdly, not only the short-term outcomes, including tumor remission and radical effect on surgery, but also the long-term outcomes, namely the survival outcomes, were rather promising.

In conclusion, the aforementioned information indicate a potential feasibility of preoperative chemotherapy for gastric cancer patients with GOO. However, there may be an underlying publication bias, and the level of the evidence available is quite low. Therefore, prospective clinical trials providing qualified evidence are required to explore the feasibility, which is precisely what the present study aimed to induce and promote.

The literature also provided other information. Firstly, a variety of chemotherapeutic agents can be applied to explore the optimal regimens of preoperative chemotherapy for gastric cancer patients with GOO. Up to nine antitumor chemicals were involved in the 11 retrieved studies. Among them, the most frequently applied agent was fluoropyrimidines. Even $\mathrm{S}-1$, with the enteral dosage form, was also medicated in 4 patients $(6-8,10)$ perhaps via a nasointestinal feeding tube in the form of a water solution. Thus, the prevailing chemotherapy regimens for gastric cancer patients currently used in clinical practice and trials can be applicable candidates for intervention measures of future clinical trials in this scope.

Secondly, nutritional support treatment may be indispensable for patients with this condition. As is well known, inadequate intake of food and water is common for patients with GOO. Thus, malnutrition and dehydration are frequently encountered by these patients. In the retrospective cohort of the analytic study aforementioned, 62 patients received preoperative chemotherapy plus concurrently administered nutritional support. The serum level of albumin and quality of life score on the last preoperative day improved. Furthermore, the extent of the improvement was more significant in the EN group compared with the PN group (5). Thus, we hypothesize that nutritional support treatment is possibly a requisite for the basis of chemotherapy implementation.

Thirdly, locally advanced (M0) gastric cancer patients may be more preferred as subjects of preoperative chemotherapy. In the retrieved studies, M0 and far advanced (M1) patients with GOO were selected as the subjects of the treatment. As for the M0 patients, preoperative chemotherapy can be deemed as a neoadjuvant treatment, while for the M1 patients it may be considered as salvage or palliative therapy. In our opinion, preoperative chemotherapy is less beneficial and less necessary for M1 patients compared with M0 patients who may be potentially curable and have more chance of radical resection from preoperative treatment, as non-surgical management for relieving GOO symptoms of M1 individuals involves stent placement, which is palliative and not applicable to locally advanced subjects. With regards to the stent, it is not recommended for application to M0 gastric cancer patients in clinical practice and trials on account of its indication for patients with short life expectancies $(28,29)$. Thus, candidates recruited in future clinical trials can be assigned as locally advanced gastric cancer patients complicated with GOO instead of far advanced patients.

Finally, the definitive roles of preoperative chemotherapy in relieving GOO and improving surgical and survival outcomes remain unclear. Among the patients involved in the referred case reports, GOO was relieved in 5 patients following preoperative chemotherapy $(8-10,12,13)$, while it remained in $2(11,14)$ and developed in $3(6,7)$. Comparative analysis concerning survival outcomes between the preoperative chemotherapy group and the surgery only group is unavailable in either the descriptive (5) or the analytic study (4). Thus, 
there is a necessity to conduct controlled studies to evaluate the virtual beneficial effect of preoperative chemotherapy for gastric cancer patients with GOO.

On account of the paucity of clinical research in this area, pilot studies prospectively conducted are in demand to explore the feasibility, safety and efficacy of preoperative chemotherapy for locally advanced gastric cancer patients with GOO. In future stage II clinical trials, which can be defined as 'feasibility studies', candidates can be allocated into the single-arm cohort without a control group. Once the feasibility is confirmed by stage II trials, stage III controlled studies with double arms can be carried out to further evaluate the short-term and long-term outcomes.

The GOO patients with distant metastasis (M1), namely far advanced cases, are usually the subjects of salvage or palliative treatment, and have no possibility or necessity of radical resection, while the M0 cases are appropriate candidates for initial surgical intervention or initial chemotherapeutic treatment. Therefore, subjects included in future trials can be assigned as locally advanced gastric cancer patients with GOO.

Intervention measures of such types of trials can be preoperative chemotherapy with prevailing regimens that are currently widely used in gastric cancer treatment. By contrast, nutritional support can be employed as a basic premise of chemotherapy. Enteral versus parenteral nutritional support can also be evaluated as to which is more beneficial to the premise of chemotherapy. However, whether EN imposes a more significant influence compared with PN in this situation remains to be studied, while $\mathrm{EN}$ has been proven to be more beneficial in other situations $(30,31)$. The initiation of the EN approach for patients with GOO varies from stent placement to nasojejunal feeding tube placement. The former is not recommended to be applied in trials of preoperative chemotherapy on account of its indication for patients with short life expectancies $(28,29)$, while the latter can be adopted to conduct EN treatment for candidates in clinical trials of preoperative chemotherapy for locally advanced gastric cancer patients with GOO.

Short-term study endpoints should also be determined. Firstly, the improvement of GOO symptoms can be employed as one of the main short-term endpoints. To determine the extent of the improvement, the level of oral intake prior and subsequent to the treatment can be measured by the Gastric Outlet Obstruction Scoring System (GOOSS) (32). The clinical success of the treatment can be defined as an ability to tolerate at least liquid food (GOOSS score 1). Secondly, radiological ORR or disease control rate (DCR) according to the Response Evaluation Criteria in Solid Tumors (RECIST 1.1) (33) based on primary tumor reduction in computed tomography scans or magnetic resonance images can also be used as a main short-term objective to evaluate the efficacy. Thus far, there is an indication from the retrieved case reports that relief of GOO symptoms was not always concomitant with radiological response $(6,11,14)$. Accordingly, a 'novel ORR' or 'novel DCR' applied to future clinical trials of preoperative chemotherapy for gastric cancer patients with GOO can be defined not only as CR plus PR, (and SD for DCR) according to the RECIST, but also as improvement of GOO symptoms. Thirdly, secondary endpoints, including resection rate or complete resection rate, histopathological tumor regression according to the Becker criteria (34), improvement of patients' pre- and post-intervention plasma albumin level, body mass index, the Eastern Cooperative Oncology Group Performance Status (also known as the WHO score) (35) and operative morbidity and mortality, can also be documented to evaluate the patients' short-term clinical outcomes.

In already published clinical trials regarding preoperative chemotherapy for gastric cancer patients, ORR varies from 27 to $74.4 \%$ (36-43), DCR from 78.9 to $100 \%(37,38,40,41)$ and $\mathrm{R} 0$ resection rate from 59 to $90.7 \%$ (36-38,40,41,43-45). In future trials on gastric cancer patients with GOO, if comparable outcomes can be achieved, it can be considered as promising results. A future treatment strategy for gastric cancer with GOO may therefore be further optimized in the field of short-term outcomes. Regardless, there is a possibility of disadvantageous results of such studies. In a multi-institutional retrospective Japanese study analyzing clinical outcomes for palliative gastrojejunostomy in unresectable advanced gastric cancer, prior chemotherapy was shown to be a significant independent predictor of poor survival (25). Although not a definitive conclusion, the result presented in the Japanese study did raise a different view in contrast to our expectation.

In conclusion, the paucity of high-quality literature in this area is perhaps the most significant finding of the literature review. Limited studies already published indicate a potential feasibility of preoperative chemotherapy for gastric cancer patients with GOO. However, the definitive role of chemotherapy as an initial treatment in relieving symptoms of GOO and improving other clinical outcomes remains unclear. Stage II and III clinical trials are expected to be conducted in order to probe into the authentic feasibility, safety and efficacy of preoperative chemotherapy for gastric cancer patients with GOO.

\section{References}

1. Cunningham D, Allum WH, Stenning SP, et al; MAGIC Trial Participants: Perioperative chemotherapy versus surgery alone for resectable gastroesophageal cancer. N Engl J Med 355: 11-20, 2006.

2. Ychou M, Boige V, Pignon JP, et al: Perioperative chemotherapy compared with surgery alone for resectable gastroesophageal adenocarcinoma: An FNCLCC and FFCD multicenter phase III trial. J Clin Oncol 29: 1715-1721, 2011.

3. Ji J: Study on Neoadjuvant Chemotherapy for Advanced Gastric Cancer[DB/OL]. http://www.clinicaltrials.gov/ct2/show/NCT0 2163291 ? cond=gastric + cancer\&intr=chemotherapy\& $\mathrm{rank}=33$. Accessed January 16, 2015.

4. Yamaguchi K, Nakagawa S, Yabusaki H and Nashimoto A: Evaluation of neoadjuvant chemotherapy with paclitaxel, 5 -fluorouracil and cisplatin for advanced gastric cancer with pyloric stenosis. Gan To Kagaku Ryoho 34: 2241-2244, 2007 (In Japanese).

5. Sun Y, Xu X, Hu J, et al: Efficacies of neoadjuvant chemotherapy plus nutritional support in advanced gastric cancer complicated with pylori obstruction. Zhonghua Yi Xue Za Zhi 94: 584-586, 2014 (In Chinese).

6. Yasuyama A, Mikata S, Soma Y, et al: Two cases of human epidermal growth factor receptor 2-positive advanced gastric cancer successfully treated with S-1, cisplatin, and trastuzumab combination therapy followed by curative resection. Gan To Kagaku Ryoho 40: 2203-2206, 2013 (In Japanese).

7. Nakayama Y, Kawasaki H, Wajima N, et al: Two cases of stage IV gastric cancer treated by resection of the primary tumor after chemotherapy combined with trastuzumab. Gan To Kagaku Ryoho 40: 2188-2190, 2013 (In Japanese).

8. Du Y, Cheng X, Xu Z, et al: D2 plus radical resection combined with perioperative chemotherapy for advanced gastric cancer with pyloric obstruction. Chin J Cancer Res 25: 479-481, 2013. 
9. Yamaguchi K, Nakagawa S, Yabusaki H and Nashimoto A A case of advanced gastric cancer responding remarkably to paclitaxel + low-dose FP therapy in a neoadjuvant setting. Gan To Kagaku Ryoho 33: 1163-1166, 2006 (In Japanese).

10. Shikano T, Koshikawa K, Kiriyama K, et al: Two cases of advanced gastric cancer with peritoneal dissemination acquired long-term response three years or more by postoperative treatment with oral anticancer drug TS-1. Gan To Kagaku Ryoho 33: 87-90, 2006 (In Japanese).

11. Sakurai K, Nashimoto A and Yabusaki H: A case of advanced gastric cancer showing pylorus stenosis with multiple liver metastases, that respond remarkably to neoadjuvant chemotherapy of combined TXL and low-dose FP. Gan To Kagaku Ryoho 30: 407-411, 2003 (In Japanese).

12. Hokita S, Takatori H, Ishigami $\mathrm{S}$, et al: A case of advanced type 4 gastric cancer with peritonitis dissemination, navel metastasis effectively treated with combined chemotherapy of biweekly paclitaxel (TXL) and TS-1. Gan To Kagaku Ryoho 30 1343-1346, 2003 (In Japanese)

13. Tanabe T, Nashimoto A, Sasaki J, et al: A case of advanced gastric cancer with abdominal para-aortic lymph node metastasis successfully treated with FLP therapy. Gan To Kagaku Ryoho 26 2073-2076, 1999 (In Japanese).

14. Iwamoto S, Kimoto M, Mure T, et al: A case of papillary adenocarcinoma of the stomach with liver metastases and carcinomatous peritonitis treated effectively by methotrexate/5-fluorouracil sequential chemotherapy. Gan To Kagaku Ryoho 24: 2005-2009, 1997 (In Japanese).

15. Castellanos AE, Stein EA, Mann BD and Podolsky ER: Gastric Outlet Obstruction[DB/OL]. http://emedicine.medscape. com/article/190621-overview2013. Accessed January 16, 2015.

16. National Center for biotechnology Information. Gastric Outlet Obstruction[DB/OL]. http://www.ncbi.nlm.nih. gov/mesh/68017219. Accessed January 16, 2015.

17. Chen JH, Wu CW, Lo SS, et al: Outcome of distal gastric cancer with pyloric stenosis after curative resection. Eur J Surg Oncol 33: 556-560, 2007.

18. Watanabe A, Maehara Y, Okuyama T, et al: Gastric carcinoma with pyloric stenosis. Surgery 123: 330-334, 1998.

19. Mackay S, Hayes T and Yeo A: Management of gastric cancer. Aust Fam Physician 35: 208-211, 2006.

20. Stupart DA, Panieri E and Dent DM: Gastrojejunostomy for gastric outlet obstruction in patients with gastric carcinoma. S Afr J Surg 44: 52-54, 2006.

21. Johnson CD and Ellis H: Gastric outlet obstruction now predicts malignancy. Br J Surg 77: 1023-1024, 1990.

22. Choi YB: Laparoscopic gatrojejunostomy for palliation of gastric outlet obstruction in unresectable gastric cancer. Surg Endosc 16 1620-1626, 2002.

23. Navarra G, Musolino C, Venneri A, et al: Palliative antecolic isoperistaltic gastrojejunostomy: A randomized controlled trial comparing open and laparoscopic approaches. Surg Endosc 20: 1831-1834, 2006

24. Nashimoto A, Yabusaki H and Nakagawa S: Treatment strategy for the type IV gastric cancer--from the standpoint of the surgery. Gan To Kagaku Ryoho 34: 983-987, 2007 (In Japanese).

25. Takeno A, Takiguchi S, Fujita J, et al; Clinical Study Group of Osaka University (CSGO), Upper GI Group: Clinical outcome and indications for palliative gastrojejunostomy in unresectable advanced gastric cancer: Multi-institutional retrospective analysis. Ann Surg Oncol 20: 3527-3533, 2013.

26. Shrikhande SV, Sirohi B, Barreto SG, et al: Indian Council of Medical Research consensus document for the management of gastric cancer. Indian J Med Paediatr Oncol 35: 239-243, 2014.

27. National Comprehensive Cancer Network: The NCCN Clinical Practice Guidelines in Oncology for Gastric Cancer (Version 1.2014) [EB/OL]. (2014.5.30) [2015.1.16]. http://www. nccn.org/professionals/physician_gls/pdf/gastric.pdf.

28. Jeurnink SM, van Eijck CH, Steyerberg EW, et al: Stent versus gastrojejunostomy for the palliation of gastric outlet obstruction: A systematic review. BMC Gastroenterol 7: 18, 2007.
29. Fiori E, Lamazza A, Demasi E, et al: Endoscopic stenting for gastric outlet obstruction in patients with unresectable antro pyloric cancer. Systematic review of the literature and final results of a prospective study. The point of view of a surgical group. Am J Surg 206: 210-217, 2013.

30. Mercadante S: Parenteral versus enteral nutrition in cancer patients: indications and practice. Support Care Cancer 6: 85-93, 1998.

31. Lindh A, Cedermark B, Blomgren $\mathrm{H}$, et al: Enteral and parenteral nutrition in anorectic patients with advanced gastrointestinal cancer. J Surg Oncol 33: 61-65, 1986.

32. Adler DG and Baron TH: Endoscopic palliation of malignant gastric outlet obstruction using self-expanding metal stents: Experience in 36 patients. Am J Gastroenterol 97: 72-78, 2002.

33. Eisenhauer EA, Therasse P, Bogaerts J, et al: New response evaluation criteria in solid tumours: revised RECIST guideline (version 1.1). Eur J Cancer 45: 228-247, 2009.

34. Becker K, Mueller JD, Schulmacher C, et al: Histomorphology and grading of regression in gastric carcinoma treated with neoadjuvant chemotherapy. Cancer 98: 1521-1530, 2003.

35. Oken MM, Creech RH, Tormey DC, et al: Toxicity and response criteria of the Eastern Cooperative Oncology Group. Am J Clin Oncol 5: 649-655, 1982

36. Tsuburaya A, Mizusawa J, Tanaka Y, et al; Stomach Cancer Study Group of the Japan Clinical Oncology Group: Neoadjuvant chemotherapy with S-1 and cisplatin followed by D2 gastrectomy with para-aortic lymph node dissection for gastric cancer with extensive lymph node metastasis. Br J Surg 101: 653-660, 2014.

37. Hirakawa M, Sato Y, Ohnuma H, et al: A phase II study of neoadjuvant combination chemotherapy with docetaxel, cisplatin, and S-1 for locally advanced resectable gastric cancer: Nucleotide excision repair (NER) as potential chemoresistance marker. Cancer Chemother Pharmacol 71: 789-797, 2013

38. Chen SS, Yang XC, Chi F, et al: A phase II study of preoperative chemotherapy with modified FOLFOX6 followed by surgery and postoperative chemoradiation in patients with localized gastric adenocarcinoma. Oncol Res 20: 327-332, 2013.

39. Sun XC, Lin J and Ju AH: Treatment of Borrmann type IV gastric cancer with a neoadjuvant chemotherapy combination of docetaxel, cisplatin and 5-fluorouracil/leucovorin. J Int Med Res 39: 2096-2102, 2011.

40. Spizzo G, Öfner D, de Vries A, et al: Preoperative chemotherapy with cisplatin and docetaxel followed by surgery and clip-oriented postoperative chemoradiation in patients with localized gastric or gastroesophageal junction adenocarcinoma: Results from a phase II feasibility study. Ann Surg Oncol 18: 677-683, 2011.

41. Li T and Chen L: Efficacy and safety of SOX regimen as neoadjuvant chemotherapy for advanced gastric cancer. Zhonghua Wei Chang Wai Ke Za Zhi 14: 104-106, 2011 (In Chinese).

42. Yoshikawa T, Omura K, Kobayashi O, et al: A phase II study of preoperative chemotherapy with S-1 plus cisplatin followed by D2/D3 gastrectomy for clinically serosa-positive gastric cancer (JACCRO GC-01 study). Eur J Surg Oncol 36: 546-551, 2010.

43. Qu JJ, Shi YR, Liu FR, et al: A clinical study of paclitaxel combined with FOLFOX4 regimen as neoadjuvant chemotherapy for advanced gastric cancer. Zhonghua Wei Chang Wai Ke Za Zhi 13: 664-667, 2010 (In Chinese).

44. Tsuburaya A, Nagata N, Cho H, Hirabayashi N, Kobayashi M, Kojima H, Munakata Y, Fukushima R, Kameda Y, Shimoda T, et al: Phase II trial of paclitaxel and cisplatin as neoadjuvant chemotherapy for locally advanced gastric cancer. Cancer Chemother Pharmacol 71: 1309-1314, 2013.

45. Biffi R, Fazio N, Luca F, et al: Surgical outcome after docetaxel-based neoadjuvant chemotherapy in locally-advanced gastric cancer. World J Gastroenterol 16: 868-874, 2010. 